\title{
A Simple, Centrifugation-Free, Sperm-Sorting Device Eliminates the Risks of Centrifugation in the Swim-Up Method While Maintaining Functional Competence and DNA Integrity of Selected Spermatozoa
}

\author{
Huidrom Yaiphaba Meitei ${ }^{1}$ Shubhashree Uppangala ${ }^{1} \cdot$ Krishna Sharan $^{2} \cdot$ Srinidhi Gururajarao Chandraguthi $^{2}$. \\ Arunkumar Radhakrishnan ${ }^{3}$. Guruprasad Kalthur ${ }^{1}$. Stefan Schlatt ${ }^{4}$ • Satish Kumar Adiga ${ }^{1}$ (D)
}

Received: 8 June 2020 / Accepted: 22 July 2020 / Published online: 30 July 2020

(C) The Author(s) 2020

\begin{abstract}
This pilot study was conducted to explore the benefits of using a centrifugation-free device based on the migration-sedimentation (MS) technique over centrifugation-based techniques in selecting competent spermatozoa, as compared with using split human semen samples. Ejaculates from 35 men undergoing semen analysis were split into four parts where one part was retained as the neat (NE) and the other three parts were subjected to sperm selection by using migration-sedimentation (MS), density gradient (DG) separation, and swim-up (SU) techniques. Sperm functional characteristics along with mitochondrial integrity, tyrosine phosphorylation, acrosome reaction, and ultrastructure were measured. The ability of selection techniques in reducing spontaneous and radiation-induced sperm DNA lesions was assessed by the TUNEL assay. In results, MS-selected spermatozoa had higher viability $(P<0.001)$, longevity in terms of total motility at the end of 6 and $18 \mathrm{~h}$ post-extraction $(P<0.001)$, and mitochondrial integrity $(P<0.001)$ compared with those selected by DG. Furthermore, spontaneous DNA lesions were significantly reduced in MS and SU fractions compared with NE $(P<0.001)$. Similarly, radiation-induced sperm DNA lesions were significantly lower in MS and SU fractions $(P<0.001)$ compared with DG. Ultrastructural analysis using scanning electron microscopy suggested a moderate, non-significant increase in the number of spermatozoa with normal head and mid-piece in MS fraction compared with other methods. In conclusion, the MS-based device offers a centrifugation-free, efficient, and reliable sperm selection method, making it suitable for partially equipped intra-uterine insemination (IUI) laboratories or office IUI programmes. Further research should focus on the safety and clinical usefulness of the device in assisted conception programmes in general and IUI in specific.
\end{abstract}

Keywords Sperm preparation $\cdot$ DNA damage $\cdot$ Migration-sedimentation $\cdot$ Sperm function $\cdot$ Swim-up $\cdot$ MIGLIS

Electronic supplementary material The online version of this article (https://doi.org/10.1007/s43032-020-00269-5) contains supplementary material, which is available to authorized users.

Satish Kumar Adiga

satish.adiga@manipal.edu

1 Department of Clinical Embryology, Kasturba Medical College, Manipal, Manipal Academy of Higher Education, Manipal 576104, India

2 Department of Radiation Oncology, Kasturba Medical College, Manipal, Manipal Academy of Higher Education, Manipal 576104, India

3 SAR Healthline Pvt. Ltd., Chennai 600010, India

4 Centre of Reproductive Medicine and Andrology, Albert-Schweitzer Campus 11, 48149 Münster, Germany

\section{Introduction}

Centrifugation-based sperm preparation techniques primarily involve centrifugal pelleting of spermatozoa in the liquified ejaculate followed by selection of healthy sperm. The swimup technique (SU) is routinely used either singly or in combination with density gradient (DG) centrifugation for the selection of the most active and motile spermatozoa [1, 2]. Both methods provide clean population of spermatozoa; however, it does not replicate the complex selection processes seen in in vivo methods. [3] Importantly, centrifugation-based techniques are reported to result in sperm damage and subsequent iatrogenic failure of pregnancy in some patients [4]. Impairment in sperm motility [5], mitochondrial damage [6], 
and DNA damage $[7,8]$ are observed with centrifugationbased sperm preparation methods. Reactive oxygen species (ROS) generated during the mechanical force of centrifugation is suggested to impair the structural and functional integrity of sperm cells $[9,10]$.

In order to prevent centrifugation-induced damage on spermatozoa, attempts are made to develop centrifugation-free devices to extract spermatozoa [11-13]. Based on the motility and by the help of gravity, sperm cells can sediment in the bottom of a tube without centrifugation. Thus, migrationsedimentation (MS) of spermatozoa are expected to avoid deleterious effects of centrifugation. A recent study has shown improved motility in the sperm fraction extracted by using the MS technique over other conventional methods [14].

MS is an old technique; $[15,16]$ however, its benefits to apply in assisted fertilization is not established with adequate fundamental scientific evidence. Due to rising concerns about the iatrogenic damage to spermatozoa, here, we explored the benefits of the centrifugation-free MS technique using a simple commercially available device for use in assisted fertilization programmes. We sought to determine if the MS method improves the selection of functionally competent, morphologically normal, and DNA-intact spermatozoa over standard centrifugation-based techniques in split human semen samples. A commercial kit designed on the migrationsedimentation principle was used in the study along with other conventional separation techniques.

\section{Materials and Methods}

\section{Study Subjects}

This pilot study included patients $(N=35)$ undergoing semen analysis as a part of infertility workup at the university infertility clinic. Institutional Ethical Committee approval was obtained (IEC 689/2018) before the initiation of the study. The patients who agreed to participate and signed the informed consent were included. Depending on the semen characteristics [17], only normozoospermic ejaculates with a minimum of 80 million total sperm number were included. Semen characteristics of the study subjects are shown in Table 1. Postsemen analysis, left over samples were split into four parts where one part was retained as neat (NE) and other three parts were subjected to sperm selection using DG separation, MS, and SU. The recovery rate was calculated as described earlier [18].

Out of 35 samples, 15 samples were X-irradiated in the radiation facility to induce DNA fragmentation in spermatozoa in order to understand the efficiency of selection techniques in eliminating DNA-fragmented spermatozoa. Irradiation of liquefied ejaculate on a petri dish was performed immediately (within $10 \mathrm{~min}$ ) using a linear accelerator (Versa
Table 1 Age and semen profile of the study subjects

\begin{tabular}{lc}
\hline & Mean $\pm \operatorname{SEM}(N=35)$ \\
\hline Patient age (in year) & $36.4 \pm 0.7$ \\
Semen volume $(\mathrm{mL})$ & $3.3 \pm 0.2$ \\
Sperm concentration $\left(10^{6} / \mathrm{mL}\right)$ & $63.1 \pm 4$ \\
Total sperm number $\left(10^{6} /\right.$ ejaculate $)$ & $208.7 \pm 20.6$ \\
Total motility $(\%)$ & $67.5 \pm 2.1$ \\
Progressive motility $(\%)$ & $44.2 \pm 1.8$ \\
Normal forms $(\%)$ & $12.7 \pm 2.2$ \\
Viability $(\%)$ & $61.9 \pm 2.1$ \\
\hline
\end{tabular}

HD, Elekta, 2013) with X-ray energy of $6 \mathrm{MV}$ at a dose rate of 5 Gy per minute. A total of 10 Gy was delivered onto the ejaculate at room temperature.

\section{Migration-Sedimentation-Based Device}

The device used in this study works on the principle of MS that was originally developed by Tea et al. [19] The device is made up of transparent bio-compatible Cyclo olefin polymer developed by Menicon Life Science, Japan. The device has four parts comprising of an outer lid, inner lid, spacer, and base as shown in Fig. 1a. The base consists of a central tube inserted inside a tubular container, which has a petal-shaped opening. According to the manufacturer, the liquefied semen/ diluted sample (maximum of $3 \mathrm{~mL}$ ) is deposited into the outer well (sample injection space) of the base, and sperm wash medium is added into the central tube after placing the inner lid until it overflows and covers the ejaculate. During incubation, only motile spermatozoa will jump over the edge of the central tube, and the sediment was at the bottom of the central tube as shown in the Fig. 1b. The petal-shaped opening increases the surface area and helps in sedimentation of more motile spermatozoa. The spacer is helpful while using lowvolume ejaculates. The inner lid prevents the mixing of immotile sperm and debris with motile spermatozoa due to convection.

\section{Sperm Selection by Using the MS Device}

One part of the split fraction was diluted up to $1.5 \mathrm{~mL}$ using sperm wash media (Earle's balanced salt solution, Cat. No. M5017, Sigma-Aldrich, USA) supplemented with sodium bicarbonate (Cat. No. S5761, Sigma-Aldrich, USA) and 0.1\% bovine serum albumin (BSA) and dispensed into the outer well after placing the spacer inside the base. Then, the inner lid was placed onto the diluted semen. After ensuring that the inner lid was properly fixed into the grove of the base, $2 \mathrm{~mL}$ of pre-warmed sperm wash media was dispensed into the central tube of the base so that the semen was completely covered 
(a)

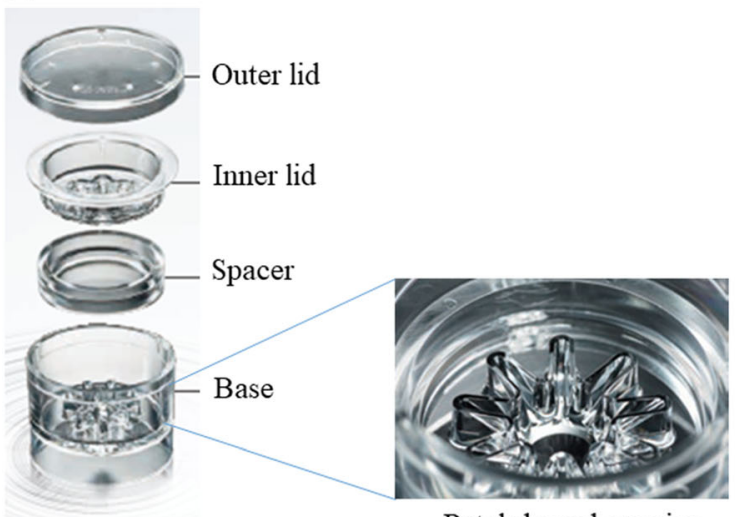

Petal shaped opening

Fig. 1 Migration-sedimentation device. a Photograph showing various parts of the device. b Schematic drawing of the device demonstrating the sedimentation of motile spermatozoa into the central tube indicated by the

with sperm wash media. The outer lid was placed on the base, and the entire unit was incubated at $37{ }^{\circ} \mathrm{C}$ and $5 \% \mathrm{CO}_{2}$. After $1 \mathrm{~h}, 0.4 \mathrm{~mL}$ of the sperm suspension was collected from the central tube to use for further analysis.

\section{Sperm Selection by Using DG}

The split fraction was overlaid on the $90 \%$ and $45 \%$ gradient solution layers (Sil-Select Plus, Cat. No. SIP100, FertiPro N.V., Belgium) and then centrifuged at $500 \mathrm{~g}$ for $20 \mathrm{~min}$. The pellet was re-suspended in $1 \mathrm{~mL}$ of pre-warmed EBSS medium supplemented with $0.1 \%$ BSA and centrifuged at $300 \mathrm{~g}$ for $8 \mathrm{~min}$. Additional washing was performed at $200 \mathrm{~g}$ for $8 \mathrm{~min}$ before the pellet was suspended in $0.4 \mathrm{~mL}$ of EBSS media supplemented with sodium bicarbonate and $0.1 \%$ BSA.

\section{Sperm Selection by Using SU}

Split fraction was mixed with $1 \mathrm{~mL}$ of pre-warmed EBSS medium supplemented with $0.1 \%$ BSA and centrifuged at $300 \mathrm{~g}$ for $8 \mathrm{~min}$. Additional washing was performed at $200 \mathrm{~g}$ for $8 \mathrm{~min}$ before the pellet was overlaid using $0.4 \mathrm{~mL}$ of EBSS media supplemented with sodium bicarbonate and $0.1 \%$ BSA. The tube was positioned at a $45^{\circ}$ angle and incubated at $37^{\circ} \mathrm{C}$ and $5 \% \mathrm{CO}_{2}$. After $1 \mathrm{~h}, 0.4 \mathrm{~mL}$ of the sperm suspension was collected to use for further analysis.

\section{Mitochondrial Membrane Potential}

Mitochondrial membrane potential was evaluated in spermatozoa as described earlier [20] with minor modifications. Approximately 0.5 million spermatozoa were incubated with $10 \mu \mathrm{g} / \mathrm{mL}$ of rhodamine 123 (Cat. No. R8004, Sigma-Aldrich, USA) at $37^{\circ} \mathrm{C}$ and $5 \% \mathrm{CO}_{2}$ for $20 \mathrm{~min}$. After washing, a drop of sperm suspension was placed on a glass slide and observed under fluorescent microscope (Imager-A1; Zeiss, Gottingen, (b)

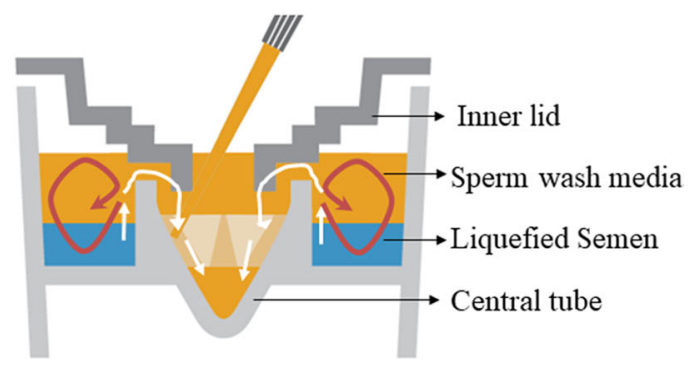

white arrow. Non-motile spermatozoa, debris, and round cells would stay in the outer well (red arrow)

Germany). Spermatozoa exhibiting bright fluorescence only at the mid-piece region was considered to have intact mitochondria (Supplementary figure 1). A minimum of 500 spermatozoa were scored from each sample, and results are expressed as the percentage of spermatozoa with damaged mitochondria.

\section{Sperm Viability}

Viability was determined by using the eosin-nigrosine staining method [17]. One drop of sperm sample was mixed with 2 drops each of $1 \%$ eosin Y and $10 \%$ nigrosin solutions; after thorough mixing, a smear was made on a pre-cleaned glass slide and air-dried. Viability was assessed using a compound microscope at $\times 100$ magnification. A minimum of 200 spermatozoa were counted to determine the percentage of viable cells. Viable spermatozoa appear unstained, whereas nonviable cells appear pink in colour.

\section{Tyrosine Phosphorylation}

Tyrosine phosphorylation in spermatozoa was determined by immunofluorescence as previously published [21] with minor modifications. Spermatozoa from each group were fixed in chilled methanol:acetone $(1: 1)$ for $10 \mathrm{~min}$ at $-20{ }^{\circ} \mathrm{C}$ followed by blocking using $1 \%$ BSA and air-dried. Cells were washed in phosphate buffered saline (PBS) and incubated with mouse monoclonal 4G10® Platinum, Anti-Phosphotyrosine Antibody, 1:200 dilution (Cat. No. 05-1050X, SigmaAldrich, USA), overnight at $4{ }^{\circ} \mathrm{C}$ in a moist chamber. Cells were washed in PBS followed by incubation with FITCconjugated goat anti-mouse IgG secondary antibody 1:1000 dilution (Cat. No. NB7538, Novus Biologicals, USA) for $30 \mathrm{~min}$ at $4{ }^{\circ} \mathrm{C}$. Counterstaining was done with $4^{\prime}, 6-$ diamidino-2-phenylindole (DAPI; $2 \mu \mathrm{g} / \mathrm{mL}$; Cat. No. D9542, Sigma-Aldrich, USA). The spermatozoa were 
mounted using Dako mounting medium (Cat. No. S3023, DAKO) and observed under fluorescence microscope at $x$ 100. A minimum of 200 cells were scored to calculate the percentage of spermatozoa displaying high-intensity fluorescence at the various regions of spermatozoa (acrosome cap, equatorial region, mid-piece, and tail) indicating the extent of sperm capacitation (Supplementary figure 2).

\section{Acrosome Reaction}

The ability of spermatozoa to undergo acrosome reaction was assessed by previously described calcium ionophore (A23187)-induced acrosome reaction (CIAR) assay [22] with minor modifications. Sperm suspension was treated with or without $5 \mu \mathrm{M}$ calcium ionophore (Cat. No. C7522, A23187, Sigma-Aldrich, USA) for $30 \mathrm{~min}$ at $37{ }^{\circ} \mathrm{C}$ and $5 \% \mathrm{CO}_{2}$. Postincubation, washed cells were smeared on the coverslip permeabilized in $100 \%$ cold methanol. Cells were stained with FITC-conjugated Pisum sativum agglutinin (FITC PSA; Cat. No. L0770, Sigma-Aldrich, USA) at a concentration of $25 \mu \mathrm{g} /$ $\mathrm{mL}$ at room temperature for $30 \mathrm{~min}$. Washed sperm cells were then counterstained with $7 \mu \mathrm{g} / \mathrm{mL}$ of propidium iodide (PI; P4170, Sigma-Aldrich, USA) and mounted on a clean slide using Dako mounting medium. A minimum of 500 spermatozoa were evaluated under a fluorescence microscope (ImagerA1; Zeiss, Gottingen, Germany). Acrosome-reacted spermatozoa had no green acrosome cap (Supplementary figure 3).

\section{Terminal Deoxynucleotidyl Transferase dUTP Nick End Labelling Assay}

Terminal deoxynucleotidyl transferase dUTP nick end labelling (TUNEL) assay was performed as per our previously published protocol [23]. Concisely, sperm cells were fixed on a poly-l-lysine-coated slide using $4 \%$ paraformaldehyde for $1 \mathrm{~h}$ and then permeabilized by $0.1 \%$ Triton X-100 in $0.1 \%$ sodium citrate solution in PBS. After 1-h equilibration at room temperature, cells were incubated with TUNEL reaction mixture (Cat. No. 12156792910, Roche Diagnostics, USA) at $37^{\circ} \mathrm{C}$ for $1 \mathrm{~h}$ in dark. The cells were washed and stained with $2 \mu \mathrm{g} / \mathrm{mL}$ DAPI (P4170, Sigma-Aldrich, USA) for $1 \mathrm{~min}$ and mounted on a clean slide. The cells were observed under a fluorescence microscope (Imager-A1; Zeiss, Gottingen, Germany) at $\times 40$ magnification. The nucleus of the TUNEL-labelled spermatozoa displayed red fluorescence (Supplementary figure 4). A minimum of 1000 spermatozoa were scored, and the labelling index was determined.

\section{Shorr Staining}

Sample containing approximately 0.1 million spermatozoa was smeared, air-dried, and stained as per the WHO protocol [17]. The stained slides were mounted using DPX and observed at $\times 100$ magnification under oil immersion. A minimum of 100 cells were assessed, and the percentage of morphological variations were recorded.

\section{Scanning Electron Microscopy}

Ultrastructural sperm morphology was analyzed using scanning electron microscopy (SEM) as described earlier [24] with minor modifications. Briefly, sperm cells were centrifuged and fixed on a clean slide using $2.5 \%$ glutaraldehyde in $0.1 \mathrm{M}$ sodium acetate buffer $(\mathrm{pH} 7.3)$ for $1 \mathrm{~h}$. The fixed cells were washed using $0.1 \mathrm{M}$ sodium acetate and subsequently dehydrated using increasing concentration of ethanol (30,50, $70,80,90$, and $100 \%$ for 10 min each). Following dehydration, the cells were air-dried and subjected to critical point drying using carbon dioxide. Finally, the cells were sputtered using gold and observed under field emission SEM (FESEM; Carl Zeiss Sigma, Germany) at $\times 20,000$ magnification. A minimum of 100 cells were analyzed for each data point as per the previously published criteria [24].

\section{Statistical Analysis}

The statistical significance for the variables was calculated by using either repeated measures analysis of variance (ANOVA) for normal distribution or Friedman's test as a nonparametric method in case failing the normality test. The statistical tests were done using the GraphPad InStat 3.0 statistical package (GraphPad Inc., USA). All the graphs were plotted using Origin 8.0 (Origin Lab Corporation, Northampton, MA, USA). $P<0.01$ was considered statistically significant.

\section{Results}

Ejaculate fractions containing equal numbers of spermatozoa were subjected to three sperm selection techniques. The total motile sperm (TMS) was comparable between MS and SU, whereas DG had a marginally lower TMS (Fig. 2a). The values for TMS and recovery rate are shown in Table 2. The recovery rate was found to be maximum in MS $(48.5 \pm 2.7)$ compared with SU $(43.6 \pm 2.9)$ and DG $(34.9 \pm 3.1)$. However, the difference was significantly different only between DG and MS $(P<0.001)$. On the other hand, viability was significantly higher in MS- $(66.4 \pm 2.1)$ and SU- $(71.6 \pm$ 2.8) extracted spermatozoa compared with that of DG (46.6 \pm $2.9 ; P<0.001$ ) (Fig. 2b). When the longevity (in terms of total motility) of the processed spermatozoa was assessed at the end of $6 \mathrm{~h}$, spermatozoa extracted by both MS and SU retained approximately $65 \%$ motility, which was significantly higher than DG $(37.9 \pm 3.4 ; P<0.001)$. A similar trend was observed at the end of $18 \mathrm{~h}$ where only about half of the spermatozoa 
(a)

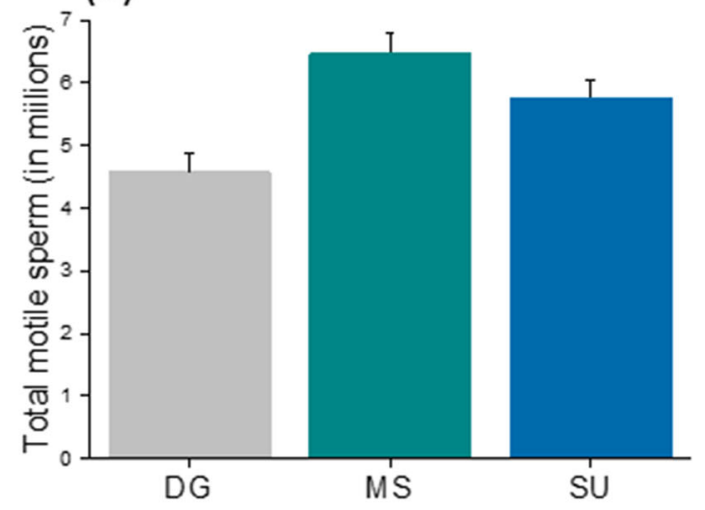

(c)

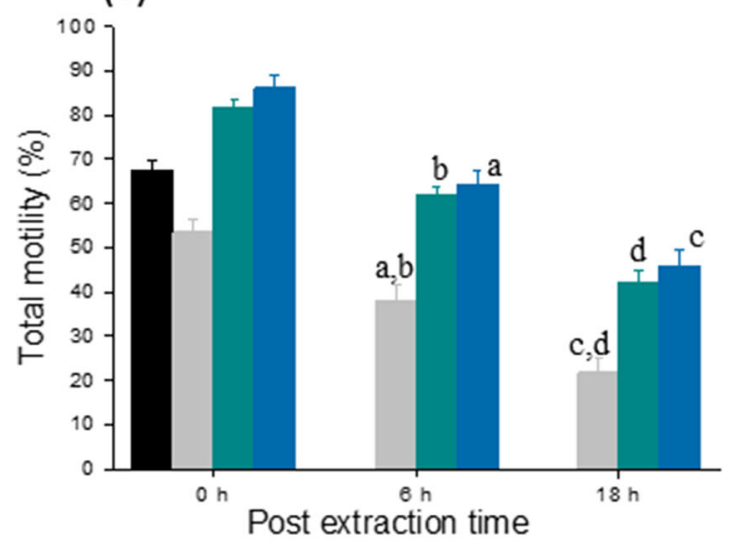

Fig. 2 Sperm functional characteristics in the processed fraction. a Total motile sperm (TMS in millions). Please note that the values are not significantly different between the groups. b Sperm viability across various groups. $P<0.001$ across identical letters $(\mathrm{a}, \mathrm{b})$. $\mathbf{c}$ Total motility immediately after selection and after 6 and 18 h. $P<0.001$ across

processed by DG showed motility $(21.6 \pm 3.2)$ significantly lower than MS $(42.2 \pm 2.6)$ and $\mathrm{SU}(45.9 \pm 3.4)$ groups $(P<0.001)$ (Fig. 2c). The mitochondrial function of the spermatozoa is important for sperm motility and longevity, and

Table 2 Total motile sperm and recovery rate observed across three study groups

\begin{tabular}{|c|c|c|c|c|}
\hline \multirow[t]{2}{*}{ Parameter } & \multirow[t]{2}{*}{ Pre-wash } & \multicolumn{3}{|c|}{ Sperm selection methods } \\
\hline & & DG & MS & SU \\
\hline Motility (\%) & $67.3 \pm 2.1$ & $53.4 \pm 2.9^{\mathrm{a}, \mathrm{b}}$ & $81.6 \pm 1.6$ & $86 \pm 2.9$ \\
\hline TMS $\left(\times 10^{6}\right.$ sperm $)$ & $13.4 \pm 0.4$ & $4.5 \pm 0.2$ & $6.4 \pm 0.3$ & $5.7 \pm 0.2$ \\
\hline Recovery rate $(\%)$ & NA & $34.9 \pm 3.1^{\mathrm{a}}$ & $48.5 \pm 2.7$ & $43.6 \pm 2.9$ \\
\hline
\end{tabular}

$T M S$, total motile sperm; $D G$, density gradient; $M S$, migrationsedimentation; $S U$, swim-up; $N A$, not applicable

All values are presented as mean \pm SEM

${ }^{\mathrm{a}} P<0.001$ vs $\mathrm{MS}$

${ }^{\mathrm{b}} P<0.001$ vs SU (b)

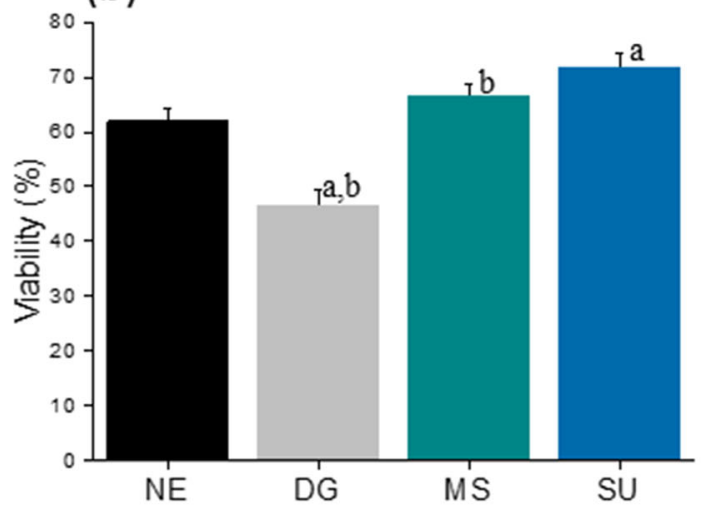

(d)

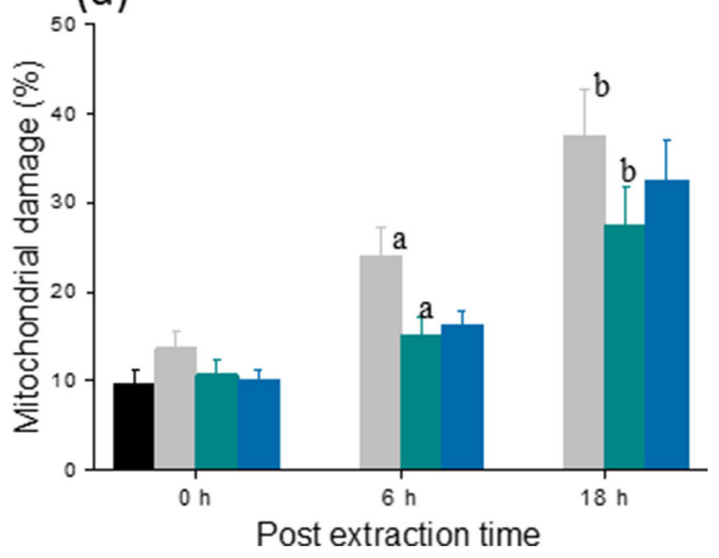

identical letters (a, b, c, d) in the same group. d Percent mitochondrial damage at various post-selection time periods. $P<0.001$ between the groups with the letter ' $a, b$ '. The bar represents mean, and the error bar represents standard error of the mean (SEM)

was assessed at different time points. Spermatozoa isolated by MS had significantly lower number of damaged mitochondria at the end of 6 and $18 \mathrm{~h}(15.06 \pm 2.0$ and 27.46 \pm 4.4 respectively) compared with those by other groups; however, statistically, a significant difference was found only with DG at 6 and $18 \mathrm{~h}(24.13 \pm 3.1$ and $37.6 \pm 5.1 ; P<0.001)$ (Fig. $2 \mathrm{~d})$.

The functional competence of extracted spermatozoa was assessed for capacitation and acrosome reaction. Number of spermatozoa displaying protein tyrosine phosphorylation, an indicator for capacitation, was significantly higher in MS $(27.2 \pm 5.3)$ and $\mathrm{SU}(26.7 \pm 6.1)$ fractions compared with $\mathrm{NE}$ $(P<0.001)$, whereas DG $(16.8 \pm 4.3)$ had lower number of positive sperm compared with MS and SU; however, the differences were not significant (Fig. 3a). On the other hand, CIAR assay demonstrated comparable number of acrosomereacted spermatozoa across all three groups (Fig. 3b).

In order to test the ability of MS in extracting DNA-intact spermatozoa, two different approaches were attempted. Initially, spermatozoa from all three methods were evaluated for TUNEL and compared with that of NE. The TUNEL index 
(a)

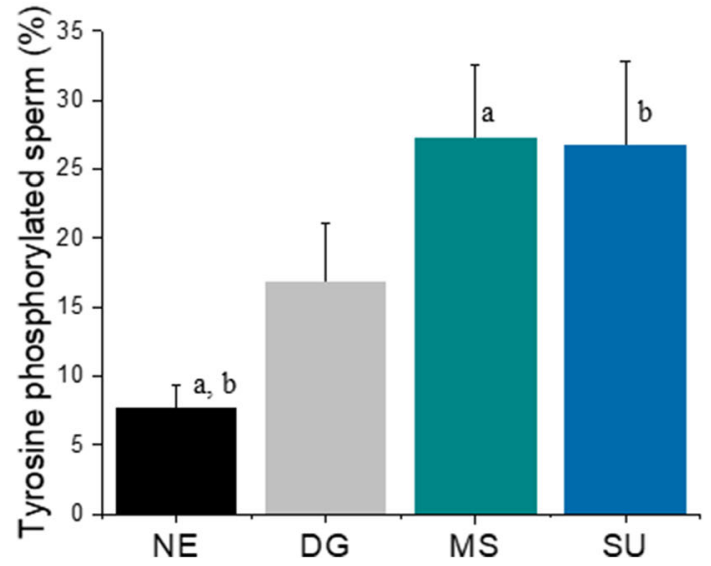

Fig. 3 a Immunostaining of spermatozoa displaying tyrosine phosphorylation. $P<0.001$ between the groups with the identical letters $(\mathrm{a}, \mathrm{b})$. b Acrosome-reacted spermatozoa. Red bar: spontaneously reached in corresponding groups; grey bar: DG; teal bar: MS; blue bar: SU

in spermatozoa post-MS and -SU was reduced to half in relation to NE $(P<0.001)$. Interestingly, a moderate but nonsignificant reduction in labelling index was observed in DGprocessed sperm fraction in comparison with NE (Fig. 4a). Alternately, spermatozoa carrying radiation-induced DNA lesions (IDL) with approximately double the number of TUNEL-positive cells $(33.3 \pm 1.9 ; P<0.001$ vs NE) were subjected to DG, MS, and SU methods to understand the efficiency of these techniques when DNA damage level is high in the ejaculates. The labelling index in DG, MS, and SU reduced significantly post-selection $(26.1 \pm 2.2,19.5 \pm 1.7$, and $18.5 \pm$ 1.5 respectively; $P<0.001$ ), whereas labelling index in MS and SU further demonstrated a reduction compared with DGextracted spermatozoa $(P<0.001)$ (Fig. $4 \mathrm{~b})$.

Results from the above experiments so far have suggested that spermatozoa extracted by MS and SU are comparable

(a)

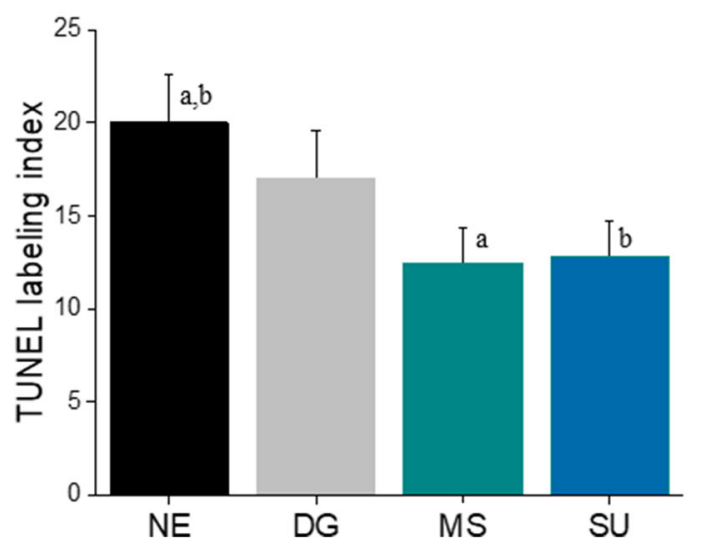

Fig. 4 Sperm DNA fragmentation in the processed fraction. a The ability of various techniques in eliminating spontaneous DNA fragmentation from the ejaculate. Black bar: NE; grey bar: DG; teal bar: MS; blue bar: SU. $P<0.001$ between the groups with the identical letters $(a, b) . b$ The (b)

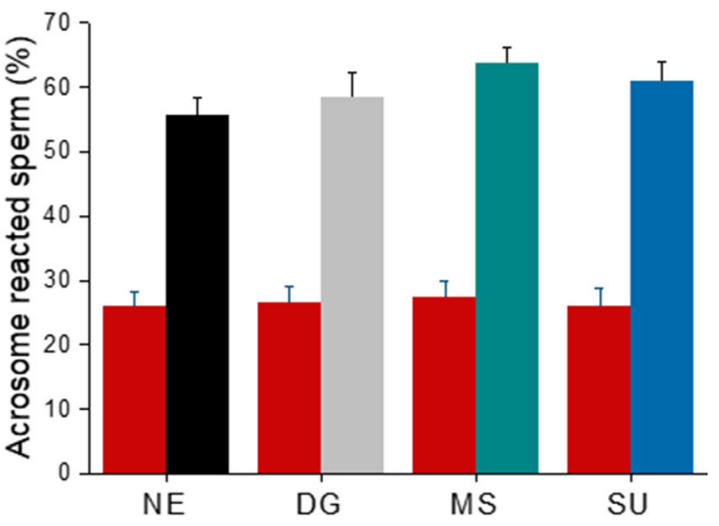

calcium ionophore-induced acrosome reaction (CIAR) in spermatozoa. Please note that the values are not significantly different between the groups. The bar and error bar represent mean and the standard error of the mean (SEM) respectively

with respect to total motile sperm, functional ability, and DNA integrity. On the other hand, DG-extracted sperm quality was found inferior to MS and SU fractions. Since MS is a non-centrifugation-based technique, we speculated that morphology of spermatozoa extracted by using this technique is inferior to other two methods as centrifugation alone or in combination with gradients, as SU and DG techniques have the ability to reduce morphologically abnormal spermatozoa. Morphology analysis of extracted spermatozoa by using the Shorr technique did not show significant variation across the three methods, though DG and MS methods have extracted moderately higher number of spermatozoa with normal head morphology, but the differences were not statistically significant (Supplementary figure 5). Furthermore, ultrastructural analysis using scanning electron microscopy also confirmed a moderate increase in the number of spermatozoa with

(b)

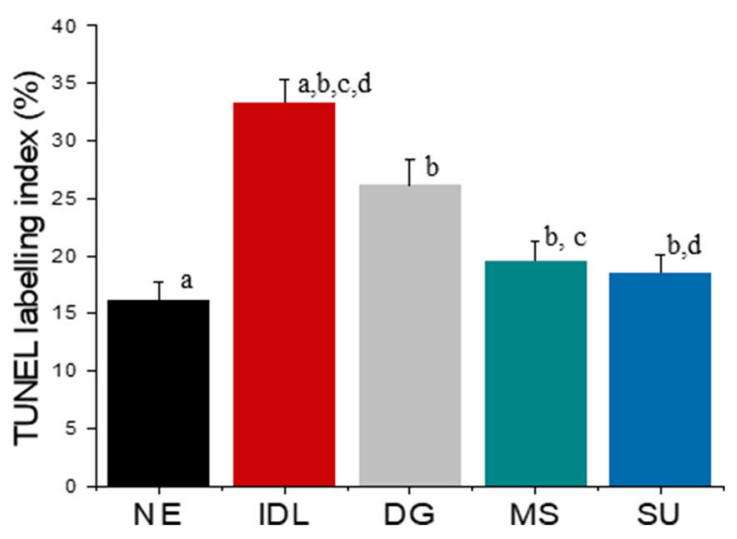

ability of various techniques in eliminating radiation-induced DNA fragmentation from the ejaculate. $P<0.001$ between the groups with the identical letters $(a, b, c, d)$. Data were presented as mean \pm SEM 
normal head and mid-piece in the MS group (Fig. 5a-e). On the other hand, amorphous forms and other mid-piece abnormalities were marginally higher in SU-extracted spermatozoa (Fig. 5b-f). However, no statistical power was demonstrated between any of these groups.

\section{Discussion}

We demonstrate that the MS-based device used for the ejaculate processing allows the selection of clinically usable, functionally competent spermatozoa with a significant reduction in the levels of DNA fragmentation. All functional parameters tested using split fractions of the ejaculates have shown that the sperm fraction can be enriched similar to the SU technique. On the other hand, density gradient centrifugation resulted in reduced motility, mitochondrial damage, and less number of capacitated spermatozoa in the processed fraction compared with MS and SU. Furthermore, the ability of DG to eliminate the number of spermatozoa carrying IDL was significantly lower than those of MS and SU methods.

MS-based sperm processing offers an alternative to traditional sperm selection techniques wherein enriched fraction of spermatozoa is isolated from an unprocessed ejaculate [15, 16]. The MS device MIGLIS $®$, a commercial product from Menicon Life Science, Japan, was used in this study. The readily available disposable device is relatively inexpensive and intended to use without the need for special technical skills or equipment, hence best suited for sperm preparation for IUI. Apart from the improvement in motility and DNA integrity via processing by using a MS device, the TMS was also comparable to other two techniques, reflecting the (a)

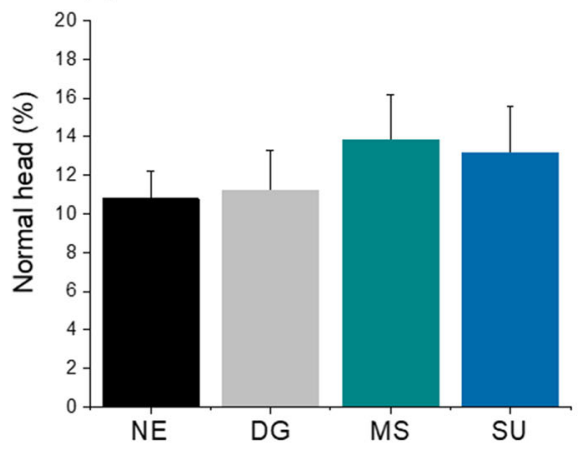

(d)

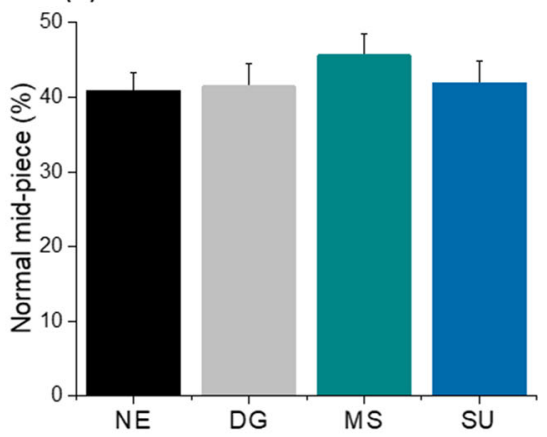

(b)

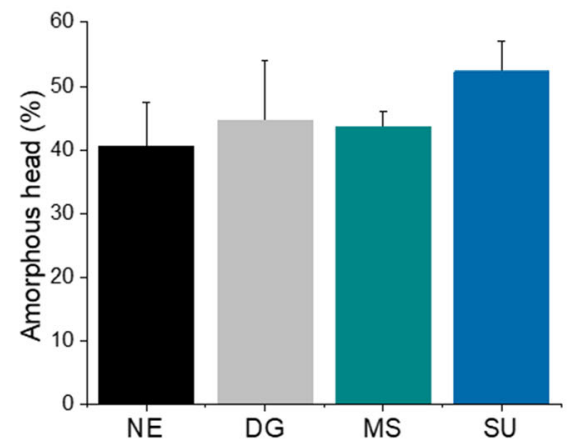

(e)

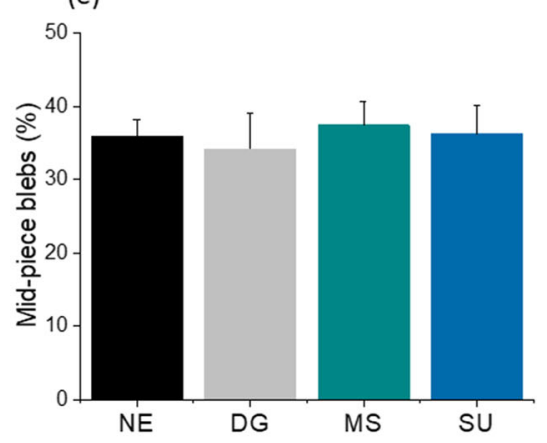

(c)

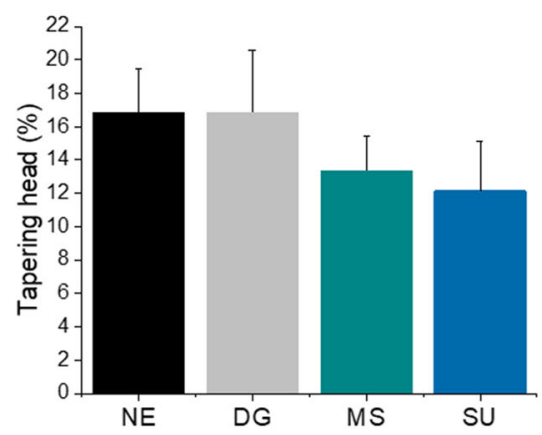

(f)

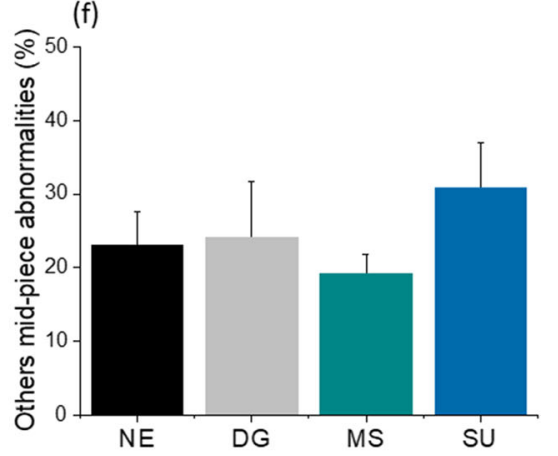

(g)

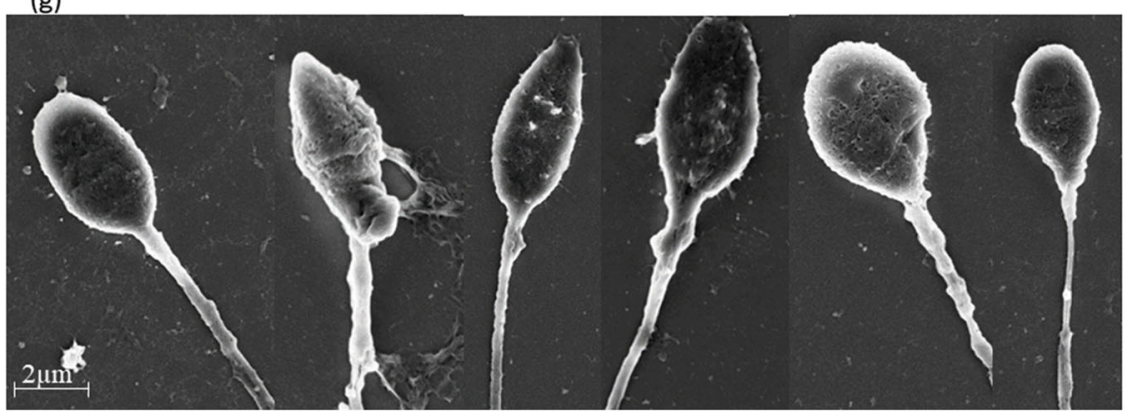

Fig. 5 Ultrastructural analysis of processed sperm fraction. $\mathbf{a}-\mathbf{c}$ Various forms of head abnormalities observed under scanning electron microscope (SEM). d-f Various mid-piece abnormalities found in the processed fraction. g Representative SEM images showing normal and abnormal forms. Please note that the values are not significantly different between the groups 
selective nature of the device without compromising the recovery rate. In our study, only split samples were processed to understand the relative benefit of the techniques employed. Since the TMS is $>5$ million and the recovery rate is approximately 50 in MS, it has potential to extract adequate number of spermatozoa to establish pregnancy in IUI programmes. Nonetheless, a single MS device is capable of processing an ejaculate up to $3 \mathrm{~mL}$, which is higher than those of the published centrifugation-free devices [11, 12], and therefore, will maximize the sperm recovery, which can be used for IUI.

Protein tyrosine phosphorylation is a key intracellular signalling event regulating sperm function and is an indicator of capacitation and essential for sperm to correctly undergo acrosome reaction. [25-27] Seminal fluid-derived proteins attached to the sperm surface can inhibit the process of capacitation by negatively affecting the hyperactive motility and protein tyrosine phosphorylation [28]. The seminal plasma was in contact with spermatozoa throughout the MS selection, and our results have shown that the protein tyrosine phosphorylation level was affected in the processed fraction; however, the level was significantly higher than that of the DG group. Furthermore, sperm motility at 6 and $18 \mathrm{~h}$ post-selection was significantly higher in MS when compared with that of DG, suggesting that exposure of seminal plasma and seminal proteins to spermatozoa during MS selection has not compromised the functionality.

Semen processing using the gradient centrifugation and swim-up procedure in this study took 40 and $80 \mathrm{~min}$ respectively, whereas the MS device needed $65 \mathrm{~min}$ to complete the process in a single step without any centrifugation. Reducing the treatment time and eliminating the centrifugation step may prevent iatrogenic damage to sperm functional and genetic integrity [4]. Though few studies have suggested that centrifugation of semen samples is associated with the generation of increased reactive oxygen species and a high DNA damage [8], the others failed to show such association [18, 29]. These conflicting observations may be related to the extent of DNA fragmentation in sperm cells, duration of the ejaculatory abstinence, quality of the ejaculate, and/or the technique used for sperm selection [30-32]. The swim-up method significantly reduces sperm DNA fragmentation rates and may have prognostic value on IUI in patients with decreased sperm DNA integrity $[8,33]$, which is in agreement with our observation. However, $\mathrm{SU}$ is relatively time-consuming, is associated with sperm seminal plasma exposure during centrifugation, and needs several rounds of centrifugation steps. On the other hand, semen processing by DG centrifugation did not improve sperm apoptotic DNA fragmentation rate [34]. Importantly, a recent study has evaluated the efficacy of SU and DG in removing DNA-damaged spermatozoa and found increased sperm DNA damage during DG and SU in few patients [35]. Our results also did not show any benefits of DG in reducing the DNA-fragmented sperm from both spontaneous and IDL-carrying ejaculates. Though, several techniques exist to test sperm DNA fragmentation such as the sperm chromatin structure assay (SCSA), the sperm chromatin dispersion (SCD) test, the TUNEL assay, and the single-cell gel electrophoresis (Comet) assay, the TUNEL assay and the Comet assay have shown better predictive value [36]. Since the TUNEL test is technically simple with less statistical robustness [37], we have employed the TUNEL assay in this study. DNA damage might be a result of mitochondrial impairment following an apoptotic cascade, [38] and motile spermatozoa can reach the bottom of the conical tube even in the presence of mitochondrial impairment and DNA damage during DG [39]. This observation is in agreement with our results, where both mitochondrial and DNA damages were higher in DGthan in MS-extracted spermatozoa. At this juncture, we would like to state that mitochondrial membrane potential measured using rhodamine 123 is not a sensitive marker, hence it is worth looking into the mitochondrial respiration by studying mitochondria complexes [40].

Based on the results observed in our study, we argue that the MS-based device used here is significantly more effective than the DG device in regard to enrichment of functional and genetic integrity in the processed fraction. Though the functional ability and genetic integrity of MS- and SU-selected spermatozoa were comparable, due to involvement of multiple centrifugation steps, SU-processed spermatozoa are at increased risk of experiencing iatrogenic damage [41]. Thus, the MS method which involves just two pipetting steps is considerably more efficient than the DG and SU techniques.

Ultrastructural analysis of spermatozoa provides a valuable tool to address subtle changes in sperm morphology which cannot be identified by using conventional staining techniques. It has been shown that the artefacts of sperm ultrastructural morphology may be associated with sperm structural fragility and preparation conditions. Spermatozoa from fertile males with centrifugation of $600 \mathrm{~g}$ for washing sperm exhibited more damage to the mid-piece than those with the $300 g$ [42]. The centrifugation speed employed in the present study $(500 \times g$ and $300 \times g$ ) is moderately higher than the WHO [17] recommendations of $300-400 \times g / 15-30 \mathrm{~min}$ for the initial wash and $200 \times \mathrm{g} /$ 4-10 min for subsequent washes in DG. Similarly, the SU technique involved two rounds of centrifugation washing instead of single-step washing and overlying followed by others. Hence, it is possible that one of the reasons for seeing increased ultrastructural changes in the mid-piece of the SU fraction could be attributed to the centrifugation steps. However, we could not establish any statistical differences in these observations possibly due to limited sample size.

Though several other non-centrifugation techniques based on microfluidic and chemotaxis principles are used for the selection of clinically usable, highly motile sperm with nearly undetectable levels of DNA fragmentation [11-13], only intracytoplasmic sperm injection (ICSI) is the possible way of fertilization due to relatively small number of spermatozoa collected at the end. IUI 
is useful only when total motile sperm count (TMSC) is $>5$ million [43], and our results have shown that MS can easily extract a minimum of 5 million TMSC from the normozoospermic split fractions. However, at this juncture, it is to be noted here with great emphasis that a major limitation of this study is that only normozoospermic ejaculates were used, as a consequence of which, the study cannot reveal the ability of the MS device in extracting adequate number of enriched spermatozoa from the poor-quality ejaculates. In this study, the EBSS + sodium bicarbonate buffering system was used in all three techniques to make the observations comparable. However, use of $\mathrm{CO}_{2}$-free incubation is the most practical approach in a physician's office or in a limited-resource setting which can be accomplished by optimizing the medium buffering system (MOPS or HEPES) and a simple dry bath incubation.

In conclusion, the MS-based device offers a centrifugationfree, efficient, and reliable sperm selection method, hence suitable for partially equipped intra-uterine insemination (IUI) laboratories or office IUI programmes. Further research should focus on the safety and clinical usefulness of the device in medically assisted conception programmes in general and IUI in specific.

Acknowledgements Open access funding provided by Manipal Academy of Higher Education, Manipal.

Authors' Contribution Conceived and designed the experiments: SKA. Performed the experiments: HYM, SU, and SGC. Analyzed the data: HYM and SU. Wrote the paper: SKA, SS, SU, GK, HYM, KS, and AR. HYM is the guarantor of this work and, as such, had full access to all the data and takes responsibility for the integrity of the data and the accuracy of the data analysis.

Funding Information HYM was the recipent of Dr. TMA Pai Doctoral fellowship from Manipal Academy of Higher Education, Manipal.

Data Availability The data and material that support the findings of this study are available from the corresponding author upon request.

\section{Compliance with Ethical Standards}

Conflict of Interest The migration-sedimentation (MS) device MIGLIS ${ }^{\circledR}$ used in this study was supplied free of cost from SAR Healthline, India.

Ethics Approval The study was approved by the Institutional Ethics committee (IEC) of Kasturba Hospital, Manipal Academy of Higher Education.

Consent to Participate Informed consent was obtained from all individual participants included in the study.

Consent to Publish Patients signed informed consent regarding publishing their data.

Open Access This article is licensed under a Creative Commons Attribution 4.0 International License, which permits use, sharing, adaptation, distribution and reproduction in any medium or format, as long as you give appropriate credit to the original author(s) and the source, provide a link to the Creative Commons licence, and indicate if changes were made. The images or other third party material in this article are included in the article's Creative Commons licence, unless indicated otherwise in a credit line to the material. If material is not included in the article's Creative Commons licence and your intended use is not permitted by statutory regulation or exceeds the permitted use, you will need to obtain permission directly from the copyright holder. To view a copy of this licence, visit http://creativecommons.org/licenses/by/4.0/.

\section{References}

1. Mortimer D. Sperm recovery techniques to maximize fertilizing capacity. Reprod Fertil Dev. 1994;6(1):25-31.

2. Volpes A, Sammartano F, Rizzari S, Gullo S, Marino A, Allegra A. The pellet swim-up is the best technique for sperm preparation during in vitro fertilization procedures. J Assist Reprod Genet. 2016;33(6):765-70.

3. Vaughan DA, Sakkas D. Sperm selection methods in the 21 st century. Biol Reprod. 2019;101(6):1076-82.

4. Mortimer D. Sperm preparation techniques and iatrogenic failures of in-vitro fertilization. Hum Reprod. 1991;6(2):173-6.

5. Matás C, Decuadro G, Martínez-Miró S, Gadea J. Evaluation of a cushioned method for centrifugation and processing for freezing boar semen. Theriogenology. 2007;67(5):1087-91.

6. Amiri I, Ghorbani M, Heshmati S. Comparison of the DNA fragmentation and the sperm parameters after processing by the density gradient and the swim up methods. J Clin Diagn Res. 2012;6(9): 1451-3.

7. Zalata A, Hafez T, Comhaire F. Evaluation of the role of reactive oxygen species in male infertility. Hum Reprod. 1995;10(6):1444 51.

8. Zini A, Finelli A, Phang D, Jarvi K. Influence of semen processing technique on human sperm DNA integrity. Urology. 2000;56(6): 1081-4.

9. Aitken RJ, Clarkson JS. Significance of reactive oxygen species and antioxidants in defining the efficacy of sperm preparation techniques. J Androl. 1988;9(6):367-76.

10. Lopes S, Jurisicova A, Sun JG, Casper RF. Reactive oxygen species: potential cause for DNA fragmentation in human spermatozoa. Hum Reprod. 1998;13(4):896-900.

11. Shirota K, Yotsumoto F, Itoh H, Obama H, Hidaka N, Nakajima K, et al. Separation efficiency of a microfluidic sperm sorter to minimize sperm DNA damage. Fertil Steril. 2016;105(2):315-21.

12. Quinn MM, Jalalian L, Ribeiro S, Ona K, Demirci U, Cedars MI, et al. Microfluidic sorting selects sperm for clinical use with reduced DNA damage compared to density gradient centrifugation with swim-up in split semen samples. Hum Reprod. 2018;33(8): 1388-93.

13. Berendsen JTW, Kruit SA, Atak N, Willink E, Segerink LI. Flowfree microfluidic device for quantifying chemotaxis in spermatozoa. Anal Chem. 2020;92(4):3302-6.

14. Kiratli S, Yuncu M, Kose K, Ozkavukcu S. A comparative evaluation of migration sedimentation method for sperm preparation. Syst Biol Reprod Med. 2018;64(2):122-9.

15. Lucena E, Lucena C, Gómez M, Ortiz JA, Ruiz J, Arango A, et al. Recovery of motile sperm using the migration-sedimentation technique in an in-vitro fertilization-embryo transfer programme. Hum Reprod. 1989;4(2):163-5.

16. Hauser R, Homonnai ZT, Paz GF, Yavetz H, Amit A, Lessing JB, et al. Migration sedimentation technique as a predictive test for the 
fertilizing capacity of spermatozoa in an in-vitro fertilization programme. Int J Androl. 1992;15(6):498-503.

17. World Health Organization, Department of Reproductive Health and Research. WHO laboratory manual for the examination and processing of human semen. Fifth edn. 2010.

18. Malvezzi H, Sharma R, Agarwal A, Abuzenadah AM, Abu-Elmagd M. Sperm quality after density gradient centrifugation with three commercially available media: a controlled trial. Reprod Biol Endocrinol. 2014;12:121.

19. Tea NT, Jondet M, Scholler R. A 'migration-gravity sedimentation' method for collecting motile spermatozoa from human semen. In: Harrison RF, Bonnar J, Thompson W, editors. In vitro fertiliz tion, embryo transfer and early pregnancy. Studies in Fertility and Sterility, vol. 1. Berlin: Springer-Dordrecht; 1984. p. 117-20.

20. Johnson LV, Walsh ML, Chen LB. Localization of mitochondria in living cells with rhodamine 123. Proc Natl Acad Sci U S A. 1980;77(2):990-4.

21. Sati L, Cayli S, Delpiano E, Sakkas D, Huszar G. The pattern of tyrosine phosphorylation in human sperm in response to binding to zona pellucida or hyaluronic acid. Reprod Sci. 2014;21(5):573-81.

22. Liu DY, Baker HW. Calcium ionophore-induced acrosome reaction correlates with fertilization rates in vitro in patients with teratozoospermic semen. Hum Reprod. 1998;13(4):905-10.

23. Kumar D, Salian SR, Kalthur G, Uppangala S, Kumari S, Challapalli S, et al. Semen abnormalities, sperm DNA damage and global hypermethylation in health workers occupationally exposed to ionizing radiation. PLoS One. 2013;8(7):e69927.

24. Liakatas J, Williams AE, Hargreave TB. Scoring sperm morphology using the scanning electron microscope. Fertil Steril. 1982;38(2):227-32.

25. Visconti PE, Kopf GS. Regulation of protein phosphorylation during sperm capacitation. Biol Reprod. 1998;59(1):1-6.

26. Ickowicz D, Finkelstein M, Breitbart H. Mechanism of sperm capacitation and the acrosome reaction: role of protein kinases. Asian J Androl. 2012;14(6):816-21.

27. Breitbart H, Finkelstein M. Regulation of sperm capacitation and the acrosome reaction by PIP 2 and actin modulation. Asian J Androl. 2015;17(4):597-600.

28. Hernández-Silva G, Chirinos M. Proteins from male and female reproductive tracts involved in sperm function regulation. Zygote. 2019;27(1):5-16.

29. Wang M, Sun J, Wang L, Gao X, Lu X, Wu Z, et al. Assessment of density gradient centrifugation (DGC) and sperm chromatin dispersion (SCD) measurements in couples with male factor infertility undergoing ICSI. J Assist Reprod Genet. 2014;31(12):1655-63.

30. Ghumman S, Adiga SK, Upadhya D, Kalthur G, Jayaraman V, Rao $\mathrm{SB}$, et al. Combination of swim-up and density gradient separation methods effectively eliminate DNA damaged sperm. J Turk Ger Gynecol Assoc. 2011;12(3):148-52.

31. Jayaraman V, Upadhya D, Narayan PK, Adiga SK. Sperm processing by swim-up and density gradient is effective in elimination of sperm with DNA damage. J Assist Reprod Genet. 2012;29(6):55763.

32. Uppangala S, Mathai SE, Salian SR, Kumar D, Singh VJ, D'Souza $\mathrm{F}$, et al. Sperm chromatin immaturity observed in short abstinence ejaculates affects DNA integrity and longevity in vitro. PLoS One. 2016;11(4):e0152942.

33. Oguz Y, Guler I, Erdem A, Mutlu MF, Gumuslu S, Oktem M, et al. The effect of swim-up and gradient sperm preparation techniques on deoxyribonucleic acid (DNA) fragmentation in subfertile patients. J Assist Reprod Genet. 2018;35(6):1083-9.

34. Stevanato J, Bertolla RP, Barradas V, Spaine DM, Cedenho AP, Ortiz V. Semen processing by density gradient centrifugation does not improve sperm apoptotic deoxyribonucleic acid fragmentation rates. Fertil Steril. 2008;90(3):889-90.

35. Muratori M, Tarozzi N, Carpentiero F, Danti S, Perrone FM, Cambi $\mathrm{M}$, et al. Sperm selection with density gradient centrifugation and swim up: effect on DNA fragmentation in viable spermatozoa. Sci Rep. 2019;9(1):7492.

36. Cissen M, Wely MV, Scholten I, Mansell S, Bruin JP, Mol BW, et al. Measuring sperm DNA fragmentation and clinical outcomes of medically assisted reproduction: a systematic review and metaanalysis. PLoS One. 2016;11(11):e0165125.

37. Evenson DP. The sperm chromatin structure assay $(\operatorname{SCSA}(\circledR))$ and other sperm DNA fragmentation tests for evaluation of sperm nuclear DNA integrity as related to fertility. Anim Reprod Sci. 2016;169:56-75.

38. Koppers AJ, Mitchell LA, Wang P, Lin M, Aitken RJ. Phosphoinositide 3-kinase signalling pathway involvement in a truncated apoptotic cascade associated with motility loss and oxidative DNA damage in human spermatozoa. Biochem J. 2011;436(3):687-98.

39. De Martin H, Miranda EP, Cocuzza MS, Monteleone PAA. Density gradient centrifugation and swim-up for ICSI: useful, unsafe, or just unsuitable? J Assist Reprod Genet. 2019;36(12):2421-3.

40. Rato L, Duarte AI, Tomás GD, Santos MS, Moreira PI, Socorro S, et al. Pre-diabetes alters testicular PGC1- $\alpha$ /SIRT3 axis modulating mitochondrial bioenergetics and oxidative stress. Biochim Biophys Acta. 2014;1837(3):335-44.

41. Twigg J, Irvine DS, Houston P, Fulton N, Michael L, Aitken RJ. Iatrogenic DNA damage induced in human spermatozoa during sperm preparation: protective significance of seminal plasma. Mol Hum Reprod. 1998;4(5):439-45.

42. Zhu WJ. Preparation and observation methods can produce misleading artefacts in human sperm ultrastructural morphology. Andrologia. 2018;50(7):e13043.

43. Lemmens L, Kos S, Beijer C, Braat DDM, Nelen WLDM, AMM $\mathrm{W}$, et al. Techniques used for IUI: is it time for a change? Hum Reprod. 2017;32(9):1835-45.

Publisher's Note Springer Nature remains neutral with regard to jurisdictional claims in published maps and institutional affiliations. 